\title{
A Stable Blunt Costophrenic Angle in Healthy Subjects, Associated with Dysanapsis and Airflow Limitation
}

Mariko Matsumoto-Yamazaki, Akihiko Ohwada", Satomi Shiota and Kazuhisa Takahashi

Division of Respiratory Medicine, Juntendo University Faculty of Medicine and Graduate School of Medicine, Japan

*Corresponding author: Akihiko Ohwada, Division of Respiratory Medicine, Juntendo University Faculty of Medicine and Graduate School of Medicine, 2-1-1 Hongo, Bunkyo-Ku, Tokyo 113-8421, Japan, Tel: 81-3-5802-1063; E-mail: aohwadac@gmail.com

Received date: Jul 20, 2014, Accepted date: Oct 10, 2014, Published date: Oct 15, 2014

Copyright: (c) 2014 Matsumoto-Yamazaki M, et al. This is an open-access article distributed under the terms of the Creative Commons Attribution License, which permits unrestricted use, distribution, and reproduction in any medium, provided the original author and source are credited.

\begin{abstract}
Objective: A blunt costophrenic (CP) angle in chest radiographs is known as a sign of pleural effusion, but is also observed in pulmonary emphysema. However, healthy young adult subjects with a blunt CP angle were often encountered in company annual medical examinations. The goal of the study was to evaluate radiographic measurements, spirometry, and cough symptoms in such subjects with a stable blunt CP angle obtained in a company medical check to clarify the underlining condition.
\end{abstract}

Methods: Radiographic measurements were made for the diameter of the main bronchus (MB), the tracheal width (TR), and the lung area (LA) estimated from the product of the height of the right lung and internal chest diameter in posteroanterior chest radiographs. The MB/LA and TR/LA ratios were calculated as markers of dysanapsis. An interview was performed to obtain information on cough symptoms.

Results: The MB/LA and TR/LA ratios were both significantly lower in subjects with a blunt CP angle than in controls without blunt $\mathrm{CP}$ angles. Spirometry in the subjects with blunt $\mathrm{CP}$ angles indicated that $57.2 \%$ of parameters, including FEF25-75\%, FEF75\%, FEF50\%, PEF, and FEV1/FVC, were subnormal and $84.1 \%$ of the subjects had a concave maximal expiratory flow-volume curve. These spirometric findings suggest the presence of airflow limitation involving the small airways. In correlation analyses, the MB/LA ratio was significantly associated with FEF25-75\%/FVC, PEF, and FEF50\%; and the TR/LA ratio was positively correlated with FEF25-75\%/FVC and FEF50\%. In interviews, $62.1 \%$ of subjects with a blunt CP angles stated that they had experienced cough symptoms in recent years or in the past.

Conclusion: A stable blunt CP angle is associated with dysanapsis and airflow limitation. These changes may explain the high rate of cough among subjects with a stable blunt CP angle.

Keywords: Costophrenic angle; Blunt; Chest radiograph; Dysanapsis; Airflow limitation; Concave shape of maximal expiratory flow-volume curve

\section{Introduction}

The costophrenic (CP) angle in a posteroanterior chest radiograph is a radiographic projection of the costodiaphragmatic recesses. Blunting of the $\mathrm{CP}$ angle is a sign of pleural effusion, but a blunt $\mathrm{CP}$ angle is also a radiographic finding associated with an overinflated lung, such as that seen in patients with pulmonary emphysema [1-3]. The term "dysanapsis" has been proposed to describe the disproportional growth of airways relative to lung parenchyma since early childhood $[4,5]$. Apart from the growth trajectory, it has been shown that lung volume and airway size may decline independently to progress to flow-to-volume dysanapsis in disease processes such as cystic fibrosis [6].

Japanese companies are required to conduct an annual medical examination for employees, including conventional posteroanterior chest radiography. In this examination, a blunt CP angle is sometimes seen in healthy subjects. In the current study, radiographic measurements were performed in healthy subjects with a stable blunt $\mathrm{CP}$ angle for 3 years or more who were identified in an annual medical check in order to clarify the presence of dysanapsis. Spirometry and an interview to obtain information on cough symptoms were also performed due to clarify pulmonary dysfunction related to dysanapsis.

\section{Materials and Methods}

\section{Subjects}

Digitalized images of posteroanterior chest radiographs were obtained in an annual company medical examination by the equipment of automatic exposure controlled digital radiographic system (Hitachi medical corp. Radnext) interfaced with KonicaMinolta picture archiving and communication system (PACS) and were evaluated to detect blunt $\mathrm{CP}$ angles unilaterally or bilaterally. The physicians who evaluated the chest radiographs had not been involved on the day of the annual examination and were blinded to all information except name, gender and age. Pleural effusion was ruled out based on visible pulmonary vasculature in the dorsal lung field beneath the diaphragm. Cases with a blunt $\mathrm{CP}$ angle accompanied with any pulmonary parenchymal shadow, overt pleural thickness, or massive cardiomegaly in chest films were excluded. We also excluded cases with a history of asbestos exposure, pleural effusion, or 
pneumothorax based on their medical information. Subjects with "stable" blunt CP angles were defined as cases in which this finding had been observed for at least three consecutive years without any progression. Among these subjects, those who agreed to participate in the study were selected as the study population. Each subject was prospectively interviewed with regard to cough symptoms, and radiographic and spirometric measurements were performed. Control subjects without a blunt $\mathrm{CP}$ angle who did not complain any current respiratory symptoms or repeated, nagging cough in recent years or in the past at interviews were randomly selected using annual company medical examination. Radiographic measurements were carried out for controls, but spirometry was not performed in controls at the company's request. All subjects provided informed consent and the study was approved by the institutional review board and ethics committee.

\section{Radiographic measurements}

The following measurements were obtained from posteroanterior chest radiographs [7]: main bronchus (MB) diameter, taken as the mean of measurements of the right and left bronchi $1 \mathrm{~cm}$ from the carina; mean of the tracheal width (TR) measured at 2, 4 and $6 \mathrm{~cm}$ above the carina; lung area (LA), estimated from the product of the height of the right lung (measured from the inferior border of the second rib to the highest point of the right hemidiaphragm) and the internal chest diameter (measured at the widest portion of the chest, using the internal margins of the ribs). Two ratios were calculated to define the relative airway size: the main bronchus diameter to the lung area $(\mathrm{MB} / \mathrm{LA})$, and the tracheal width to the lung area (TR/LA).

\section{Spirometry}

Spirometry was conducted with Autospiro AS-302 (Minato, Japan) according to ATS/ERS guidelines and the highest FVC, FEV1, FEV1/FVC ratio, and PEF values were taken from technically acceptable recordings $[8,9]$. FEF at 25\% (FEF25\%), 50\% (FEF50\%), 75\% (FEF75\%) of FVC expired, and FEF25-75\% were measured from the recording with the largest sum of FEV1 and FVC $[8,9]$.

These parameters are presented as absolute values and as percentiles of the predicted values (\% predicted). Spirometric reference data reported by the Japanese Respiratory Society for FVC, FEV1, FEV1/FVC, and FEF50\% [10] and reference data from elsewhere for PEF [11], FEF25-75\% [11], and FEF75\% [12] were used for interpretation of spirometric values. Thus, by convention, FVC, FEV1, FEF75\%, FEF50\%, FEF25\%, and FEF25-75\% values $<80 \%$ of the predicted value and $\mathrm{FEV} 1 / \mathrm{FVC}$ values $<70 \%$ of the predicted value were considered to be subnormal. The FEF25-75\% / FVC ratio was calculated. We also investigated the presence of a concave maximal expiratory flow-volume (MEFV) curve, which was defined as a curve with a scoop between the peak of the curve and the endpoint of expiration (Figure 1) [9].

\section{Data Analysis}

All data are presented as means \pm SD. Analyses were performed by non-parametric unpaired $t$ test (Mann-Whitney $U$ ) for comparison of radiographic measurements between control subjects and subjects with stable blunt $\mathrm{CP}$ angles. Correlation coefficients of MB/LA and TR/LA with spirometric parameters were calculated by the Pearson method. Comparison of radiographic measurements and spirometric parameters between coughers and non-coughers among the subjects with stable blunt $\mathrm{CP}$ angles were performed by non-parametric unpaired t test (Mann-Whitney $\mathrm{U}$ ). All analyses were performed using Graphpad Prism (Graphpad Software, Inc, San Diego, CA). A p value of $<0.05$ was considered to indicate a significant difference.

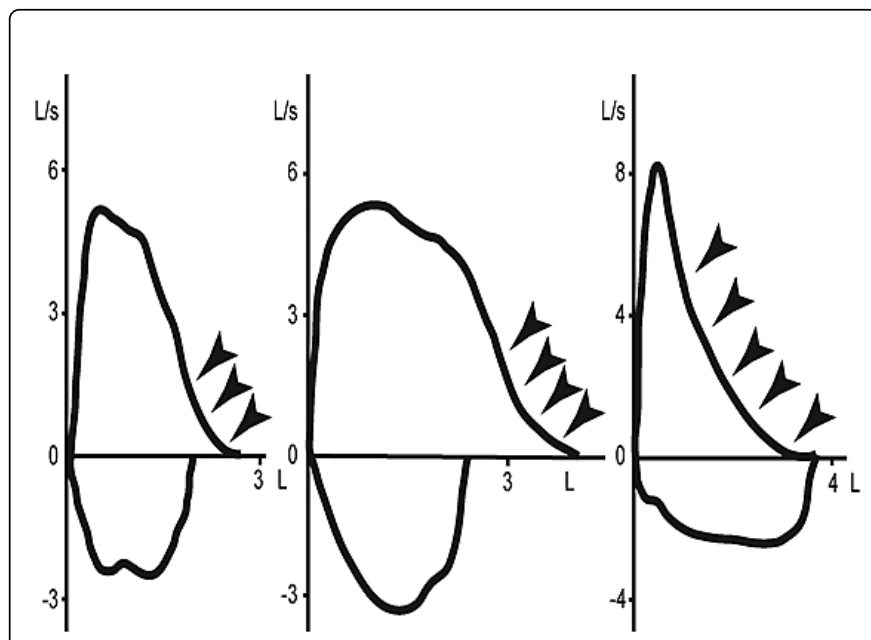

Figure 1: Representative patterns of concave maximal expiratory flow-volume curves in subjects in the study. Arrows indicate the concave region of the curve

\section{Results}

A total of 145 subjects with "stable" blunting of the CP angle (Figure 2) and 87 control subjects were selected for the study. The subjects with blunt CP angles were aged between 19 and 66 years old ( $37.7 \pm 11.2$ years), and 128 were male.

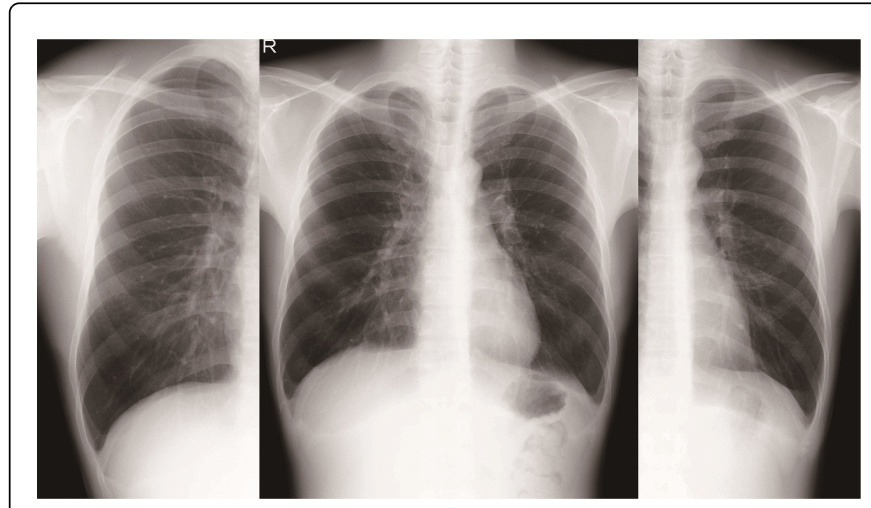

Figure 2: Blunt costophrenic angle in chest radiographs in subjects in the study. Left, male aged 27 years old (corresponding to schematic diagram $\mathrm{C}$ in Figure 4); Center, male aged 20 years old (corresponding to schematic diagram B or C in Figure 4); Right, male aged 40 years old (corresponding to schematic diagram $B$ in Figure 4).

There were 74 current smokers, 10 former smokers who had stopped smoking for over 3 years, and 61 subjects who had never smoked. Control subjects were aged between 22 and 63 years old (41.8 \pm 13.6 years), and included 57 males, 53 current smokers, 4 exsmokers, and 30 subjects who had never smoked. Of the 145 subjects 
Page 3 of 5

with blunt CP angles, 90 (62.1\%) answered that they had repeated, nagging coughs in recent years or in the past, and 9 subjects also complained of phlegm. However, their information about the severity, duration, and character of cough symptoms was obscure due to inaccuracy of their memories.

The MB/LA $\left(1.32 \times 10^{-4} \pm 0.24 \times 10^{-4}\right.$ vs. $2.42 \times 10^{-4} \pm 0.34 \times 10^{-4}$, $\mathrm{p}<0.0001)$ and TR/LA $\left(1.73 \times 10^{4} \pm 0.32 \times 10^{-4}\right.$ vs. $2.87 \times 10^{4} \pm 0.41 \times$ $\left.10^{-4}, \mathrm{p}<0.0001\right)$ ratios were significantly lower in subjects with blunt $\mathrm{CP}$ angles compared to those in control subjects (Figure 3 ).

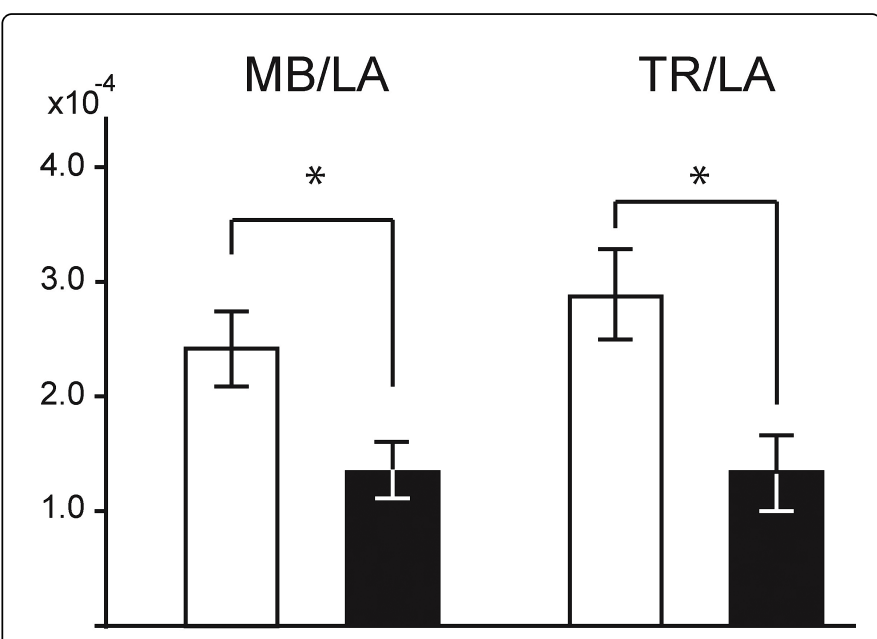

Figure 3: $\mathrm{MB} / \mathrm{LA}$ and TR/LA ratios, radiographic indexes of dysanapsis in control subjects (open column) and subjects with a blunt CP angle (solid column). Both ratios were significantly lower in subjects with a blunt $\mathrm{CP}$ angle than in control subjects. ${ }^{\star} \mathrm{p}<0.0001$ for each comparison.

The values for spirometric parameters and percentages using reference values were $5.76 \pm 0.99 \mathrm{~L}(130.7 \pm 16.96 \%$ predicted $)$ for FVC, $4.67 \pm 0.92 \mathrm{~L}(122.5 \pm 16.9 \%$ predicted $)$ for FEV1, $81.2 \pm 9.5 \%$ for FEV1/FVC, $9.16 \pm 2.09 \mathrm{~L} / \mathrm{s}$ (100.7 \pm 23 \%predicted) for PEF, $4.51 \pm$ $1.53 \mathrm{~L} / \mathrm{s}$ (90.99 $\pm 36.86 \%$ predicted) for FEF25-75\%, $5.27 \pm 1.62 \mathrm{~L} / \mathrm{s}$ $(101.8 \pm 25.3 \%$ predicted $)$ for FEF50\%, $2.30 \pm 1.03 \mathrm{~L} / \mathrm{s}(94.1 \pm 36.6$ \%predicted) for FEF75\%, and $0.79 \pm 0.25$ for the FEF25-75\%/FVC ratio. Of the 145 subjects, $83(57.2 \%)$ had at least one subnormal spirometric parameter, including 59 for FEF25-75\%, 53 for $\mathrm{FEF} 75 \%$, 28 for FEF50\%, 21 for PEF, and 18 for FEV1/FVC.

Concave MEFV curves were found in 122 subjects (84.1\%). In total, 127 of 145 subjects (87.6\%) with a blunt CP angle on a chest radiograph had a subnormal spirometric parameter or a concave MEFV curve, or both.

To explore the factors that influenced the $\mathrm{MB} / \mathrm{LA}$ and TR/LA ratios, correlations with spirometric parameters were calculated (Table 1).

\begin{tabular}{|l|l|l|l|l|}
\hline & \multicolumn{2}{l|l|l|}{ MB/LA } & \multicolumn{2}{l|}{ TR/LA } \\
\hline & $\mathrm{r}$ & $\mathrm{p}$ & $\mathrm{r}$ & $\mathrm{p}$ \\
\hline FVC \%pred. & 0.013 & 0.088 & -0.141 & 0.091 \\
\hline FEV1 \%pred. & 0.115 & 0.168 & -0.037 & 0.659 \\
\hline FEV1/FVC & 0.08 & 0.337 & 0.121 & 0.147 \\
\hline FEF25-75\% \%pred. & 0.089 & 0.285 & 0.084 & 0.315 \\
\hline
\end{tabular}

\begin{tabular}{|l|l|l|l|l|}
\hline FEF25-75\%/FVC & 0.179 & $\mathbf{0 . 0 3 1}$ & 0.252 & $\mathbf{0 . 0 0 2}$ \\
\hline PEF \%pred. & 0.173 & $\mathbf{0 . 0 3 7}$ & 0.133 & 0.111 \\
\hline FEF50\% \%pred. & 0.23 & $\mathbf{0 . 0 0 6}$ & 0.165 & $\mathbf{0 . 0 4 7}$ \\
\hline FEF75\% \%pred. & 0.14 & 0.093 & 0.137 & 0.01 \\
\hline
\end{tabular}

Table 1: Correlations of MB/LA and TR/LA with spirometric parameters

Bold text indicates a significant correlation between the parameters.

For MB/LA values, positive correlations were observed with FEF25-75\%/FVC, PEF (\%predicted) and FEF50\% (\%predicted). The TR/LA ratio was positively correlated with FEF25-75\%/FVC and FEF50\% (\%predicted).

We compared radiographic and spirometric parameters between the coughers $(n=90)$ and non-coughers $(n=55)$ among the subjects with stable blunt CP angle. The TR/LA ratio alone was statistically different $(\mathrm{p}=0.025)$ between the coughers $(1.76 \times 10-4 \pm 0.33 \times 10-4)$ and non-coughers $(1.69 \times 10-4 \pm 0.30 \times 10-4)$. Comparisons of the $\mathrm{MB} / \mathrm{LA}$ ratio and all spirometric parameters were not significantly different.

\section{Discussion}

A normal CP angle is formed by convergence of the costal line and diaphragmatic line at a single point (Figure 4A). A blunt CP angle is thus defined as that in which the costal and diaphragmatic lines do not intersect at one point and make a round or balloon shape. In the early phase of pleural fluid accumulation, initial medial displacement of the $\mathrm{CP}$ angle (separation of a sharp pulmonary angle from the inner curve of the rib) occurs without blunting (Figure 4D) [13].

This phenomenon is a result of the lung still having sufficient structural rigidity to maintain its edge, thereby forcing some of the fluid to go to the lateral side of the lung [13]. Blunting of the CP angle then develops with disease progression (Figure 4E). None of our cases had medial displacement of the $\mathrm{CP}$ angle, but had a less acute $\mathrm{CP}$ angle or a balloon shape, as shown in the schematic diagrams in Figures $4 \mathrm{~B}$ and $4 \mathrm{C}$ and the representative radiographs in Figure 2.

In previous radiographic evaluations of dysanapsis, Munakata et al. used an index calculated from the tracheal cross-sectional area divided by FVC [14], while Sheel et al. evaluated lung and airway sizes at various levels of the airway tree using three-dimensional computed tomography [15]. Using an easily applicable method based only on posteroanterior chest radiographs, Litonjua et al. [7] showed that low $\mathrm{MB} / \mathrm{LA}$ and TR/LA ratios are significant predictors of higher methacholine airway responsiveness, after the FEF25-75\%/FVC ratio. Parker et al. showed that a low FEF25-75\%/FVC ratio was associated with higher airway sensitivity and reactivity to methacholine in susceptible subjects [16], and Vilozni et al found that the FEF25-75\%/FVC ratio reflects the severity of flow-to-volume dysanapsis (regardless of airway responsiveness) and showed that a rapid decline of this ratio occurs approximately 4 years before lung transplantation in patients with cystic fibrosis [6].

In our study, the MB/LA and TR/LA ratios were both significantly related to FEF25-75\%/FVC, and FEF50\%. The normal value of the FEF25-75\%/FVC ratio is not well defined. Mirsadraee et al. found FEF25-75\%/FVC ratios of $0.90 \pm 0.24$ for normal controls, $0.73 \pm 0.19$ for subjects at risk of COPD, and $0.31 \pm 0.17$ for those with COPD 
[17]. In children (4 to 18 years of age), Drewek et al. showed that the FEF25-75\%/FVC ratio was lower in those with a positive methacholine challenge test $(0.82 \pm 0.21)$ compared to those with negative results $(0.97 \pm 0.23)$ [18]. The FEF25-75\%/FVC ratio was relatively low in our subjects.
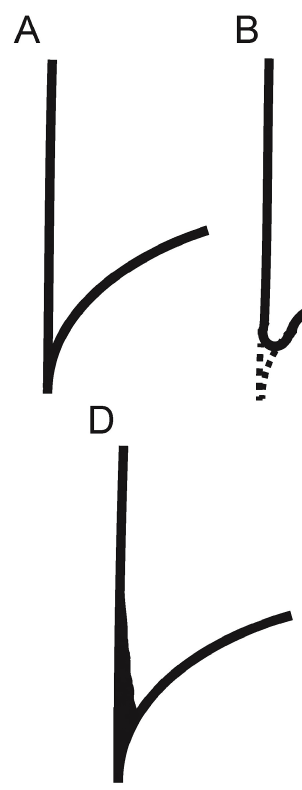

B

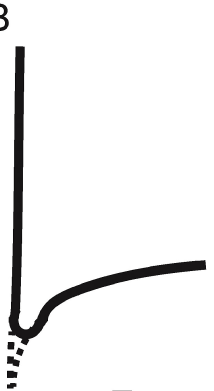

E
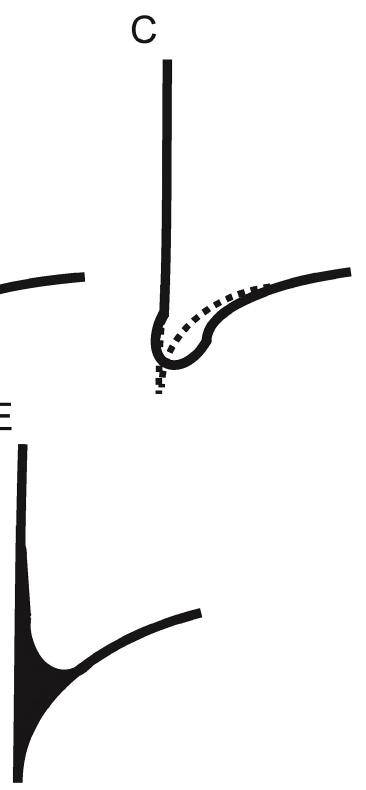

Figure 4: Schematic diagrams of the costophrenic angle. A, normal $\mathrm{CP}$ angle, defined as a case in which the costal and diaphragmatic lines intersect at one point; $\mathrm{B}$ and $\mathrm{C}$, blunt $\mathrm{CP}$ angle, defined in this study as convergence of the costal and diaphragmatic lines at a less acute angle (B) or a balloon shape (C) without medial displacement of the $\mathrm{CP}$ angle; $\mathrm{D}$, displacement of the $\mathrm{CP}$ angle from the inner curve of the rib, an early sign of pleural effusion; E, blunt CP angle due to pleural effusion, which is accompanied by medial displacement of the $\mathrm{CP}$ angle.

In spirometric analyses, subjects with a stable blunt $\mathrm{CP}$ angle had a high incidence $(57.2 \%)$ of subnormal values of spirometric parameters, especially those related to small airway dysfunction, such as FEF25-75\% FEF50\%, and FEF75\% [19]. The subjects also had a higher incidence $(84.1 \%)$ of concave MEFV curves. Many analyses of the configuration of the MEFV curve were performed in the 1970s and 1980s, with a particular focus on the concave shape. This shape is not disease specific and is observed in patients with bronchial asthma, COPD, dyspnea, and wheeze $[20,21]$. In 2005, the American Thoracic Society (ATS)/European Respiratory Society (ERS) task force [8] summarized the concave shape of the MEFV curve as follows. The earliest changes associated with airflow obstruction in small airways are thought to be slowing in the terminal portion of the spirogram, even when the initial part of the spirogram is barely affected. This slowing of expiratory flow is most obviously reflected in the concave shape of the MEFV curve, and is reflected quantitatively in a reduced FEF at $75 \%$ of FVC expired (FEF75\%) or FEF between 25 and $75 \%$ of VC (FEF25-75\%). However, an additional phrase in the final paragraph indicated that abnormalities in midrange flow measurements during forced exhalation are not specific for some cases with small airway diseases. This phrase caused a negative impression regarding use of a concave MEFV curve in practice. For this reason, we previously reconfirmed the importance of a concave MEFV curve as a sign of airflow limitation in adult asthmatic patients with physicianconfirmed wheeze and a positive response to short-acting beta agonists, and showed a tight linkage of this shape with spirometric parameters of small airway obstruction (FEF50\%, FEF75\%, and FEF25-75\%) [9].

The present study was performed as a prospective pilot study and has several limitations. These include no postbronchodilation spirometry and no spirometric evaluation of control subjects without blunt CP angles. These studies were not performed at the company's request. However, we found that subjects with stable blunt $\mathrm{CP}$ angles had significantly lower $\mathrm{MB} / \mathrm{LA}$ and TR/LA ratios compared with controls, suggesting the presence of dysanapsis. Many subjects also had subnormal spirometric parameters, including a low FEF25-75\%/FVC ratio and a concave MEFV curve, suggesting the presence of airflow limitation in small airways. A low FEF25-75\%/FVC ratio also implies the presence of airway responsiveness besides dysanapsis.

It would be criticized unsatisfactory information about cough symptoms in the subjects with stable blunt CP angle. Since they did not remember well except troubled with coughs, precise information such as frequency, intensity, character such as daytime or nocturnal cough will be obtained by precomposed cough diary in future study. In comparison of radiographic and spirometric parameters between the coughers and the non-coughers in the subjects with stable blunt $\mathrm{CP}$ angles, nothing but the values of the TR/LA ratio in the coughers were larger than those of the non-coughers against our expectations. However, it remains unclear to explain the meaning of this significant but small difference.

The exact pathophysiological basis of a blunt CP angle is unknown, but one possible explanation is that this sign represents inhomogeneity of regional ventilation because of its strong linkage with flow-tovolume dysanapsis and a concave MEFV curve $(6,20)$. Since our study population was young adults, it would be not due to destructive process of overinflation observed in pulmonary emphysema.

These underlining conditions may explain the high incidence of cough symptoms $(62.1 \%)$ among the subjects with blunt CP angles. However, further study is necessary to clarify whether the degree of either dysanapsis or airflow limitation or both influences the severity or frequency of cough symptoms

\section{Acknowledgements}

We are grateful to Dr. Katsuhiko Sato, former chief physician at the Tokyo Plant, Bridgestone Corp., for help with and management of clinical data collection.

\section{References}

1. Thurlbeck WM, Simon G (1978) Radiographic appearance of the chest in emphysema. AJR Am J Roentgenol 130: 429-440.

2. Sutinen S, Christoforidis AJ, Klugh GA, Pratt PC (1965) Roentogenologic criteria for the recognition of nonsymptomatic pulmonary emphysema. Correction between roentogenologic findings and pulmonary pathology. Am Rev Respir Dis 91: 69-76.

3. Pratt PC (1987) Role of conventional chest radiography in diagnosis and exclusion of emphysema. Am J Med 82: 998-1006. 
Citation: Matsumoto-Yamazaki M, Ohwada A, Shiota S, Takahashi K (2014) A Stable Blunt Costophrenic Angle in Healthy Subjects, Associated with Dysanapsis and Airflow Limitation. J Pulm Respir Med 4: 210. doi:10.4172/2161-105X.1000210

Page 5 of 5

4. Mead J (1980) Dysanapsis in normal lungs assessed by the relationship between maximal flow, static recoil, and vital capacity. Am Rev Respir Dis 121: 339-342.

5. Martin TR, Feldman HA, Fredberg JJ, Castile RG, Mead J, et al. (1988) Relationship between maximal expiratory flows and lung volumes in growing humans. J Appl Physiol (1985) 65: 822-828.

6. Vilozni D, Lavie M, Sarouk I, Efrati O (2012) Progressive flow-to-volume dysanapsis in cystic fibrosis: a predictor for lung transplantation? Am J Respir Crit Care Med 186: 82-87.

7. Litonjua AA, Sparrow D, Weiss ST (1999) The FEF25-75/FVC ratio is associated with methacholine airway responsiveness. The normative aging study. Am J Respir Crit Care Med 159: 1574-1579.

8. Pellegrino R, Viegi G, Brusasco V, Crapo RO, Burgos F, et al. (2005) Interpretative strategies for lung function tests. Eur Respir J 26: 948-968.

9. Ohwada A, Takahashi K (2012) Concave pattern of a maximal expiratory flow-volume curve: a sign of airflow limitation in adult bronchial asthma. Pulm Med 2012: 797495.

10. Japanese Respiratory Society (2004) Standard reference values of spirometric parameters and arterial blood gas analysis. (Author translated) in Japanese, Jap Respir J.

11. Hankinson JL, Odencrantz JR, Fedan KB (1999) Spirometric reference values from a sample of the general U.S. population. Am J Respir Crit Care Med 159: 179-187.

12. Bouhuys A (1970) Pulmonary function measurements in epidemiological studies. Bull Physiopathol Respir (Nancy) 6: 561-578.

13. Rudikoff JC (1980) Early detection of pleural fluid. Chest 77: 109-111.

14. Munakata M, Ohe M, Homma Y, Kawakami Y (1997) Pulmonary dysanapsis, methacholine airway responsiveness and sensitization to airborne antigen. Respirology 2: 113-118.
15. Sheel AW, Guenette JA, Yuan R, Holy L, Mayo JR, et al. (2009) Evidence for dysanapsis using computed tomographic imaging of the airways in older ex-smokers. J Appl Physiol (1985) 107: 1622-1628.

16. Parker AL, Abu-Hijleh M, McCool FD (2003) Ratio between forced expiratory flow between $25 \%$ and $75 \%$ of vital capacity and FVC is a determinant of airway reactivity and sensitivity to methacholine. Chest 124: 63-69.

17. Mirsadraee M, Boskabady MH, Attaran D (2013) Diagnosis of chronic obstructive pulmonary disease earlier than current Global Initiative for Obstructive Lung Disease guidelines using a feasible spirometry parameter (maximal-mid expiratory flow/forced vital capacity). Chron Respir Dis 10: 191-196.

18. Drewek R, Garber E, Stanclik S, Simpson P, Nugent M, et al. (2009) The FEF25-75 and its decline as a predictor of methacholine responsiveness in children. J Asthma 46: 375-381.

19. van der Wiel E, ten Hacken NH, Postma DS, van den Berge M (2013) Small-airways dysfunction associates with respiratory symptoms and clinical features of asthma: a systematic review. J Allergy Clin Immunol 131: 646-657.

20. Mead J (1978) Analysis of the configuration of maximum expiratory flow-volume curves. J Appl Physiol Respir Environ Exerc Physiol 44: 156-165.

21. Kapp MC, Schachter EN, Beck GJ, Maunder LR, Witek TJ Jr (1988) The shape of the maximum expiratory flow volume curve. Chest 94: 799-806. 